\title{
Los efectos de la copresencia en los aprendizajes en la escuela: la interacción en educación física en tres actividades individuales con alumnos de 10 a 11 años
}

\author{
The Effects of the Learninng in Presence at School: \\ Interaction in Physical Education in three Individual Activities \\ with Students from 10 to 11 Years Old
}

Calire Durand*

http://dx.doi.org/10.21503/CienciayDesarrollo.2008.v9.06

\section{RESUMEN}

La motivación de este trabajo de investigación ha sido describir los modos en que interactúan los participantes de una práctica motriz en el contexto del proceso de aprendizaje vinculado a la educación física, así como establecer cuáles son los efectos de tal interacción. Para el efecto, se han considerado tres formas de interacción: la de copresencia pasiva, la de copresencia activa sin interacción motriz, y la de copresencia con interacción motriz. Adicionalmente, se intenta confirmar los efectos que estas formas de interacción tienen sobre la motricidad y la socioafectividad de los sujetos involucrados en ella. La población objeto del estudio estuvo conformada por 400 niños de 10 a 11 años de edad, del nivel escolar 3.

Key words: educación fisica, actividad motriz, interacción

\section{ABSTRACT}

The motivation of this investigation work has been to describe the ways in that interactúan the participants of a motive practice in the context of the learning process linked to the physical education, as well as to settle down which the effects of such an interaction are. For the effect, they have been considered three interaction forms: that of passive copresencia, that of active copresencia without motive interaction, and that of copresencia with motive interaction. Additionally, it is tried the effects that these interaction forms have on the motricidad and the socioafectividad of the fellows involved in her to confirm. The population object of the study was conformed by 400 children from 10 to 11años of age, of the school level 3.

Key words: physical education, motive activity, interaction.

* Catedrática de Educación Física y Deporte (Institut Universitaire de Formation des Maîtres).

(C) 2008 All rights reserved.

DOI: http://dx.doi.org/10.21503/CienciayDesarrollo.2008.v9.06 


\section{INTRODUCCIÓN}

En la clase, en medio de esa agrupación de alumnos, la interacción es esencial. Con cualquier asignatura enseñada, el aprendizaje se desarrolla obviamente en situación de copresencia. Esta situación específica no deja de tener consecuencias en el aprendizaje, como lo ponen en evidencia numerosos trabajos (Bandura(1); Wynnikamen(2); Oberlé, Mugny \& Beauvois(3); Doise \& Mugny(4); Gilly, Fraisse \& Roux(5); Perret-Clermont(6); Meirieu(7)).

En el curso de educación física, fuera del marco familiar de la clase, el profesor tiene que enterarse, de manera empírica, de que esta copresencia provoca también efectos de aprendizaje.

Las diferentes tareas motrices que el profesor propone a los alumnos varían desde un punto de vista interaccional. Algunas se llevan a cabo en solitario, pues no es necesario acudir a otro para cumplir estas actividades, y el sujeto se encuentra en una situación de falta de interacción con sus compañeros. Las situaciones de aprendizaje que se asumen entonces se denominan "psicomotrices".(2)

Otras actividades solo se efectúan entre varios; en cuyo caso la interacción motriz puede incluir acciones de cooperación (con uno o varios compañeros), de oposición (con uno o varios adversarios) o a la vez con estos dos tipos de relaciones (como en los juegos deportivos colectivos). En estos casos, la interacción es constitutiva de las tareas propuestas; se evocan entonces situaciones "sociomotrices".(3) Dos grandes clases de situaciones motrices son así determinadas a partir de un criterio: la presencia o la ausencia de interacción motriz o práxica.

Este artículo da cuenta de los principales resultados y de la metodología de una experimentación llevada a cabo en el marco de una tesis de sociología (Durand(8)) cuyo objeto es estudiar, en una población de 400 sujetos de 10 a 11 años de edad y alumnos en 17 clases de nivel 3(4), los efectos diferenciales de cuatro categorías de situaciones motrices de aprendizaje practicadas en tres actividades individuales: el roller, la carrera de velocidad y el kayac (Durand(9)). Escogidas en las dos clases de situaciones evocadas anteriormente, tres categorías de situaciones son "psicomotrices": motricidad en solitario, simultaneidad (actuar alternativamente) y alternancia (actuar y observar alternativamente). La cuarta es "sociomotriz": sociomotricidad de cooperación estricta (actuar únicamente en interacción motriz de cooperación).

\section{PROBLEMÁTICA}

\section{A. Panorama de investigaciones}

Un panorama de investigaciones sobre el tema muestra resultados a veces divergentes sobre los efectos de la copresencia. Para algunos autores, la copresencia facilita los progresos motores (Triplett(10); Allport(11); Arripe(12); Fleurance \& Wynnikamen; Lafont(13)), aun cuando para otros desempeña más bien un papel de inhibición (Zajonc(14); Jackson \& Williams(15); Sanna(16)). Además, se ha identificado desde hace varias decenas de años el fenómeno de "pereza social" (Ringelman(17)).

\section{B. Los postulados teóricos de esta investigación}

Esta investigación está apoyada sobre una concepción interaccionista: el sujeto es un actor comprometido en "un sistema de acción” (Crozier, Friedeberg(18)), la identidad individual y la identidad social existen juntas (Elias(19)). En el plano de la motricidad, el sujeto que actúa está situado al centro de un sistema interaccional con los otros y con su medio físico (Parlebas(20)). 


\section{La definición del objeto de estudio}

Con el fin de evaluar algunos efectos de una copresencia organizada durante la práctica motriz por el profesor, se va a contestar la pregunta: "¿Qué modalidades relacionales o interaccionales(5) se ejecutarán entre los actores en la práctica motriz y para qué efectos?"

\section{Las hipótesis de la investigación}

\section{- Hipótesis principal}

"Las mismas prácticas individuales provocan efectos de aprendizaje diferentes según el contextorelacionalen elquese aprenden. Elgrado de esta relación: copresencia pasiva (motricidad sin interactividad con otros), copresencia activa sin interacción motriz (comotricidad en simultaneidad y en alternancia), copresencia con interacción motriz (sociomotricidad de cooperación estricta), es una variable que influye en los efectos de aprendizaje así como en la dinámica socioafectiva de los grupos de alumnos y en las opiniones de los practicantes".

\section{- Hipótesis secundarias}

Postulan efectos sobre la motricidad y la socioafectividad, así como en cuanto a las preferencias de los sujetos.

\section{METODOLOGÍA}

A. Plan de experiencia escogido para recoger las bases. Aparece en la Tabla 1 para todas las etapas experimentales.

B. Variables dependientes y tratamiento de los resultados

- Progresos motores: son evaluados en referencia con diferencias de performances y de notas de cualidad motriz entre pretest y postest motores (test de student unilateral).

- Cohesión socioafectiva: calculamos las diferencias de cohesión socioafectiva en los grupos de experiencia y de control entre precuestionariosy postcuestionarios sociométricos (indicio de cohesión socioafectiva).

- Preferencias de los alumnos sobre la modalidad relacional de práctica motriz: proponemos un cuestionario al final de la experimentación (Khi 2).

\section{RESULTADOS}

A. Todos los resultados aparecen en la Tabla 2 para cada modalidad relacional y para cada variable dependiente recogida. Para

Tabla 1. Plan típico de experiencia

\begin{tabular}{|c|c|c|c|c|c|c|}
\hline & \multicolumn{2}{|c|}{$\begin{array}{l}\text { PRIMERA FASE } \\
\qquad(1 \text { semana })\end{array}$} & \multicolumn{2}{|c|}{$\begin{array}{c}\text { SEGUNDA FASE } \\
\text { (6 semanas) }\end{array}$} & \multicolumn{2}{|c|}{$\begin{array}{l}\text { TERCERA FASE } \\
\quad(1 \text { semana })\end{array}$} \\
\hline $\begin{array}{c}\text { Grupos } \\
\text { Clases de } \\
\text { «cours } \\
\text { moyen» } \\
\mathrm{N}=17\end{array}$ & $\begin{array}{c}\text { Primer } \\
\text { cuestionario } \\
\text { de } \\
\text { sociometría } \\
\text { "Q1" }\end{array}$ & $\begin{array}{c}\text { Primera } \\
\text { prueba } \\
\text { (recorrido } \\
\text { cronometra- } \\
\text { do y filmado) } \\
\text { "P1" }\end{array}$ & $\begin{array}{c}6 \text { cursos de } 1 \text { hora efectiva } \\
\text { intercalados en una modalidad } \\
\text { idéntica para cada una de las } 3 \\
\text { actividades escogidas }\end{array}$ & $\begin{array}{c}\text { Última } \\
\text { prueba } \\
\text { (recorrido } \\
\text { cronometra- } \\
\text { do y filmado) } \\
\text { "P2" }\end{array}$ & $\begin{array}{l}\text { Segundo } \\
\text { cuestionario } \\
\text { de } \\
\text { sociometría } \\
\text { "Q2" }\end{array}$ & $\begin{array}{c}\text { Cuestionario } \\
\text { de opiniones } \\
\text { "Q3" }\end{array}$ \\
\hline
\end{tabular}


Tabla 2. Clasificación de las modalidades relacionales de aprendizaje

\begin{tabular}{|c|c|c|c|c|}
\hline & \multicolumn{2}{|c|}{ RESULTADOS MOTRICES } & \multirow{2}{*}{ Cohesión } & \multirow{2}{*}{$\begin{array}{l}\text { Opiniones } \\
\text { favorables }\end{array}$} \\
\hline & Cronometrados & Notados & & \\
\hline $\begin{array}{c}\text { Sociomotricidad coop. } \\
\text { estricta }\end{array}$ & 3ème $(n=34)$ & 3 eme $(n=29)$ & 1ere $(n=+6)$ & 1ere $(n=68 \%)$ \\
\hline Simultaneidad & 2ème $(n=20)$ & 4eme $(n=36)$ & 3eme $(n=0)$ & 3 eme $(n=53 \%)$ \\
\hline Alternancia & 1ère $(n=19)$ & 1ere $(n=16)$ & 2eme $(n=+2)$ & 2eme $(n=54 \%)$ \\
\hline En solitario & 4ème $(n=35)$ & 2eme $(n=28)$ & 3 eme $(n=0)$ & 5eme $(n=38 \%)$ \\
\hline En solit. control & Sème $(n=57)$ & 5eme $(n=56)$ & Seme $(n=-1)$ & 4eme $(n=43 \%)$ \\
\hline
\end{tabular}

Texto:

\begin{tabular}{|c|c|c|c|}
\hline $\begin{array}{l}\text { (n) Para los } \\
\text { resultados motrices: }\end{array}$ & $\begin{array}{c}\text { Notas obtenidas } \\
\text { para cada modalidad } \\
\text { relacional }\end{array}$ & & Adición algebraica del número de puntos \\
\hline (n en \%) Para las opiniones: & $\begin{array}{c}\% \text { de respuestas } \\
\text { "mucho” conseguidas } \\
\text { en el cuestionario de } \\
\text { opiniones }\end{array}$ & $\begin{array}{l}\text { (n) Para la } \\
\text { cohesión }\end{array}$ & $\begin{array}{c}\text { obtenidos en relación con los aumentos } \\
(+1) \text {, disminuciones }(-1) \text { o igualdades }(0) \\
\text { de cohesión en los grupos experimentales } \\
\text { o de control }\end{array}$ \\
\hline
\end{tabular}

compararlos, están clasificados con notas para los resultados motores y la cohesión. Para los resultados motrices, las notas más bajas corresponden a las mejores clasificaciones; para la cohesión es lo contrario.

B. Los resultados más significativos son:

- La alternancia: una modalidad eficaz para aprender.

- La sociomotricidad de cooperación: favorece la cohesión del grupo. Todos los grupos en cooperación han aumentado su cohesión al final de la experiencia.

- La simultaneidad aumenta las performances; la motricidad en solitario favorece la calidad de la realización motriz.

- En los grupos de control en solitario, se presentaron unos resultados netamente en retroceso.

- Opiniones favorables para el trabajo en cooperación (68\% de respuestas "mucho") y en comotricidad de simultaneidad y de alternancia ( $53 \%$ y $54 \%$ de respuestas "mucho").

\section{CONCLUSIONES}

Desde el punto de vista del sociólogo, nuevas investigaciones podrían interesarse en un determinado número de variables sociales que influyen en los aprendizajes en las diferentes configuraciones relacionales puestas a prueba aquí. Se propondría, entonces, cruzar, por ejemplo, diferentes tipos de agrupaciones de alumnos (según criterios de género, edad, medio sociocultural de origen, etc.).

Verdadero elemento pertinente en las tareas motrices,lamodalidadrelacionaldeprácticapuede ser controlada y manipulada por el profesor, con tal de que tenga el conocimiento de sus efectos. Los resultados de la investigación presentada en este artículo deberían ofrecer algunas pistas útiles para construir una enseñanza eficaz que descanse en un conocimiento verdadero de los efectos de un aprendizaje que se realiza necesariamente en situación de copresencia. 


\section{REFERENCIAS BILIOGRÁFICAS}

1. Bandura Albert. L'apprentissage social. Liège, Mardaga, 1986.

2. Winnykamen Faïda. Apprendre en imitant. Paris, PUF. Collection Psychologie d'aujourd'hui, 1990.

3. Oberlé Dominique, Mugny Gabriel, Beauvois Jean Léon. La psychologie sociale (I) Relations humaines, groupes et influences sociales. Presses Universitaires de Grenoble, 1995.

4. Doise Wilhem, Mugny Gabriel. Psychologie sociale et développement cognitif. Paris, Armand Colin, 1997.

5. Gilly Michel, Fraisse Jacques, Roux Jean Paúl. «Interactions entre pairs et progrès cognitifs dans trois tâches de résolution de problèmes chez des enfants de 13 ans». In: A.-N. Perret-Clermont \& M. Nicolet (Eds). Interagir et connaître: enjeux et régulations sociales dans le développement cognitif. Paris, L'Harmattan, 2001. p. 79-101.

6. Perret - Clermont Anne-Nelly. La construction de l'intelligence dans l'interaction sociale. Berne, Peter Lang, 2000.

7. Meirieu Philippe. Outils pour apprendre en groupe. Apprendre en groupe-2. Lyon, chronique sociale, 1996.

8. Durand Claire. Activités psychomotrices, apprentissages moteurs et dynamique socio-affective. Les effets de quatre modalités relationnelles de pratiques motrices en cours moyen. Th. Sociologie. Paris 5 La Sorbonne, 2006.

9. Durand Claire. «La coprésence en éducation physique et sportive: les effets de quatre modalités relationnelles de pratiques adoptées en kayak au cours moyen ». Revue STAPS $\mathrm{N}^{\circ}$ 77, 2007. p. 30-47.

10. Triplett Norman. «The dynamogenic factors in pacemaking and competition $\gg$.
Américan Journal of Psychologie, № 9, 1897. pp. 507-533.

11. Allaport F H. «The influence of the group upon association and Thought». Journal of expérimental psychologie, 1920.3, P. 159-182.

12. Arripe Fabienne (d'), Fleurance Philippe, Wynnikamen Faïda. Efficacité comparée du travail individuel et du travail en situations dyadiques sur l'apprentissage d'une habileté gymnique chez des adolescents. Congrès international de la société Française de Psychologie du sport. Poitiers, 1994. p. 217-218.

13. Lafont Lucile. Modalités sociales d'acquisition d'habiletés motrices complexes. Thèse. Psychologie (non publiée), Paris V, La Sorbonne, 1994.

14. Zajonc R B. «Social facilitation». In: Revue sciences $\mathrm{N}^{\circ} 149,1965$. p. 249-274.

15. Jackson J M, Williams K D. «Social loafting on difficult tasks: Working collectivety can improve performance». Journal of personality and social psychology, $\mathrm{N}^{\circ} 49,1985$. $\mathrm{p}$. 937-942.

16. Sanna L J. «Self-efficacity theory». Journal of personality and social psychology, $\mathrm{N}^{\circ}$ 62, 1992.

17. Ringelmann Maximilien. «Recherches sur les moteurs animés: travail de l'homme». Annales de l'Institut National Agronomique, 1913. 2ème series, 12, p.1-40.

18. Crozier Michel, Friedelberg Erhard. L'acteur et le systeme. Les contraintes de l'action collective. Paris, Seuil, 2ème édition. 1981.

19. Elías Norbert. La société des individus. Paris, Fayard, 1991.

20. Parlebas Pierre. Contribution à un lexique commenté en science de l'action motrice. Paris, INSEP, 1981.

21. Parlebas Pierre. Jeux, sports et sociétés. Lexique de praxéologie motrice. Paris, PUF, Collection recherche INSEP, 1999. 